\title{
miR-29b regulates Ang II-induced EMT of rat renal tubular epithelial cells via targeting PI3K/AKT signaling pathway
}

\author{
HONGTAO HU ${ }^{1}$, SHUANG HU ${ }^{1}$, SHEN XU ${ }^{1}$, YUE GAO $^{1}$, FANG ZENG $^{1,2}$ and HUA SHUI ${ }^{1}$ \\ ${ }^{1}$ Department of Nephrology, Zhongnan Hospital of Wuhan University, Wuhan, Hubei 430071; \\ ${ }^{2}$ Department of Nephrology, Ganzhou People's Hospital, Ganzhou, Jiangxi 341000, P.R. China
}

Received December 19, 2016; Accepted February 13, 2018

DOI: $10.3892 /$ ijmm.2018.3579

\begin{abstract}
Renal interstitial fibrosis is a necessary step in the progression of chronic kidney to end stage renal disease. MicroRNA-29 (miR-29) has been shown to play essential roles in epithelial-mesenchymal transition (EMT), and thus may contribute to the regulation of renal interstitial fibrosis. However, the role of miR-29 in the regulation of EMT during chronic kidney disease and renal transplantation has been a source of intense debate, and the mechanisms underlying this process are incompletely understood. In this study, we investigated the function of miR-29b in the regulation of EMT and to gain a better understanding of the mechanism by which miR-29b modulates EMT by targeting the phosphatidylinositol 3-kinase/protein kinase B (PI3K/AKT) signaling pathway during the process of renal interstitial fibrosis. The rat proximal tubular epithelial cell line NRK-52E was cultured in DMEM and treated with angiotensin II (Ang II) at various concentrations. RT-PCR was performed to investigate changes in the levels of expression of miR-29b in NRK-52E cells and western blotting was used to analyze the expression of PI3K, p-AKT, vimentin and keratin 18 . The result of the study show that treatment of NRK-52E cells with Ang II induced the transition of the cellular phenotype from epithelial to mesenchymal and upregulated the PI3K/AKT signaling pathway; this was also found following treatment with a phosphatase and tensin homolog on chromosome 10 (PTEN)-specific inhibitor. Increased expression of miR-29b was able to reverse the phenotype induced by Ang II in NRK-52E cells and blocking miR-29b activity with an miR-29b inhibitor resulted in enhanced EMT. Additionally, the PI3K/AKT
\end{abstract}

Correspondence to: Dr Hua Shui, Department of Nephrology, Zhongnan Hospital of Wuhan University, 169 Donghu Road, Wuhan, Hubei 430071, P.R. China

E-mail: shuihua2005@aliyun.com

Abbrebiations: EMT, epithelial-mesenchymal transition

Key words: epithelial-mesenchymal transition, microRNA-29b, RIF, phosphatidylinositol 3-kinase/protein kinase B, signaling pathway, phosphatase and tensin homolog on chromosome 10 , SF1670 signaling pathway was found to be suppressed in the presence of enhanced expression of miR-29b by direct binding to 3'-untranslated region (3'-UTR) of PIK3R2. We concluded that miR-29b plays an important role in the negative regulation of Ang II-induced EMT via PI3K/AKT signaling pathway and propose that enhancing miR-29b level or blocking PI3K/AKT signaling pathway may be a novel therapeutic target in renal interstitial fibrosis.

\section{Introduction}

Renal interstitial fibrosis is the compulsory route for chronic kidney disease (CKD) progressing to end-stage renal disease (ESRD), and is closely related to the loss of renal function. Its main pathological features are the multiplication of fibroblast and the accumulation of extracellular matrix, which leads to the formation of interstitial fibrosis. More and more studies have demonstrated that epithelial-mesenchymal transition (EMT) of renal tubular epithelial cell is one of the important mechanisms of multiplication of renal tubular fibroblasts (1). So exploring the molecular mechanism of EMT is important to delay the process of renal interstitial fibrosis, to search effective prevention and control measures and to prolong the life of patients. However, the mechanism of the intracellular signaling pathways involved in EMT were complex and incompletely understood.

EMT, a process characterized by downregulating epithelial characteristics and acquiring mesenchymal characteristics, is essential in development, wound healing and stem cell behaviour, and contributes pathologically to fibrosis and cancer progression. In the past several years, EMT has emerged as an important pathway leading to generation of matrix-producing fibroblasts and myofibroblasts in diseased kidney (2), but the signaling pathway underlying this process are poorly understood.

Phosphatidylinositol3-kinase/proteinkinaseB(PI3K/AKT) pathway plays a central role in cell growth, survival, and metabolism. The function of PI3K is to catalyze the cellar phosphatidylinositol(3,4,5)-trisphosphate [PtdIns(3,4,5)P3], which can phosphorylate AKT, one of the key pathways downstream of PI3K (3). Phosphatase and tensin homolog on chromosome 10 (PTEN), a phosphatidylinositol 3'-phosphatase that converts $\operatorname{PtdIns}(3,4,5) \mathrm{P} 3$ to phosphatidylinositol 4,5-bisphosphate, has been for a long time 
attributed to its lipid phosphatase activity against $\mathrm{PI}(3,4,5) \mathrm{P} 3$, the phospholipid product of the class I PI3Ks (4). Activation of PI3K/AKT/mTOR signaling contributes to the pathogenesis of many tumor types (5). Some recent studies indicated that it also acts as an vital mediator in organ fibrosis, including pulmonary fibrosis (6), liver fibrosis (7) and myocardial fibrosis. A recent study demonstrated that fluorofenidone inhibits nicotinamide adeninedinucleotide phosphate oxidase via PI3K/AKT pathway in the pathogenesis of renal interstitial fibrosis, suggesting that PI3K/AKT is involved in the EMT in the process of interstitial fibrosis (8).

Angiotensin II (Ang II), the main effector of the reninangiotensin system, contributes to the development of renal fibrosis by inducing epithelial-mesenchymal transition (9). Binding to Ang II type 1 receptor (AT1R), Ang II may modulate the ECM deposition in NRK-52E via complex intracellular signalling pathways including stimulation of PI3K/AKT (8). Moreover, the multiple effects mediated by Ang II require a dynamic modulation of gene expression/protein translation, which is in part under the control of microRNAs (miRNAs).

MicroRNAs (miRNAs) are endogenous small non-coding RNAs that regulate gene expression by degrading target mRNA or suppressing their translation at the 3 -untranslated regions (3'-UTR). During recent decades, the understandings of miRNAs on molecular mechanisms in various disease processes are expanding rapidly $(10,11)$. miR-29b, a member of miRNAs family, also exert great influence on the pathological process. Increasing evidence showed that miR-29b may act as an important pathogenic modulator in fibrosis in organs, such as heart (12), lung (13) and liver (7).

In spite of these preliminary studies, there are few reports regarding the role that the PI3K/AKT pathway plays in the Ang II induced-EMT. Thus, further investigation of miR-29b regulation in renal tubular epithelial cell is essential to uncover the molecular mechanism underpinning EMT. In the present study, we demonstrated the ability of miR-29b to prevent EMT development in renal tubular epithelial cell via repressing $\mathrm{PI} 3 \mathrm{~K} / \mathrm{AKT}$ signaling pathway.

\section{Materials and methods}

Cell culture. The normal rat kidney epithelial cell line NRK-52E was purchased from American Type Culture Collection (CRL-1571; Manassas, VA, USA). The NRK-52E cells were cultured in Dulbecco's modified Eagle's medium (DMEM) (low glucose) containing 5\% fetal bovine serum (FBS) (both from Gibco-BRL, Gaithersburg, MD, USA) and $1 \%$ streptomycin (100 $\mu \mathrm{g} / \mathrm{ml}$; Invitrogen, Carlsbad, CA, USA) and penicillin $(100 \mathrm{U} / \mathrm{ml})$ and incubated in a humidified atmosphere of $95 \% \mathrm{O}_{2}, 5 \% \mathrm{CO}_{2}$ at $37^{\circ} \mathrm{C}$ in a $\mathrm{CO}_{2}$ incubator. The cells were seeded on 6-well culture plates to $60-70 \%$ confluence in the complete medium containing $10 \%$ FBS for $24 \mathrm{~h}$. One day before treatment, cells were incubated with serum-free media for $24 \mathrm{~h}$ to synchronize the cell growth. When the cells grew to $90 \%$ confluency, they were then harvested by a brief exposure to $0.05 \%$ trypsin-EDTA (Gibco-BRL) and passaged every 2 days.

Treatments and groups. Medium was changed every two days to maintain sufficient nutrient and then the cells were treated with Ang II at different concentrations (10, 100 and 1,000 nM) for $24 \mathrm{~h}$. SF1670 group was treated with SF1670 at the concentration of 100, 500 and 1,000 $\mathrm{nM}$ for $60 \mathrm{~min}$. Untreated cells served as the control and were cultured in the same condition. The following experiments were carried out after the cells were cultured at $37^{\circ} \mathrm{C}$ in an incubator containing $5 \% \mathrm{CO}_{2}$ for 24 or $48 \mathrm{~h}$

Western blot analysis. Cells were washed with cold phosphate-bovine serum (PBS) and lysed with RIPA lysis buffer (Beyotime, Shanghai, China) containing protease and a phosphatase inhibitor (both from Roche, Mannheim, Germany). Bradford Protein Assay (Beyotime) was performed to measure the total amount of protein content. Total cell lysates were separated by $12 \%$ sodium dodecyl sulfate-polyacrylamide gel electrophoresis and transferred on to a polyvinylidene difluoride (PVDF) membrane. Non-specific binding was blocked with 5\% fatty-free milk for $2 \mathrm{~h}$ at room temperature and the membranes were incubated overnight at $4^{\circ} \mathrm{C}$ with primary antibodies against PI3K, p-AKT, vimentin and cytokeratin 18 (Cell Signaling Technology, Danvers, MA, USA) followed by incubation with secondary antibodies for at least $1 \mathrm{~h}$ at room temperature. The membrane was then washed three times in TBS containing $0.1 \%$ Tween- 20 for $10 \mathrm{~min}$. The antibodies were detected using a horseradish peroxidase linked secondary antibody (Santa Cruz Biotechnology, Inc., Santa Cruz, CA, USA) and the ECL Western Blotting Detection system (Amersham, Buckinghamshire, UK). The membrane was re-blotted with an anti-actin antibody to verify equal loading of the protein in each lane.

Quantitative real-time polymerase chain reaction. Total RNA from NRK-52E cells was extracted using TRIzol reagent (Invitrogen) according to the manufacturer's protocol. After determining the purity and quality of the total RNA, miR-29b was quantified by two step real-time PCR by using the miScript-reverse transcription kit and the miRNA-SYBR-Green kit (Qiagen, Valencia, CA, USA) according to the manufacturer's recommendations. The PCR reaction was performed using the ABI 7500 Fast Real-Time PCR system (Applied Biosystems, Bedford, MA, USA). The amplification program was $95^{\circ} \mathrm{C}$ for $15 \mathrm{~min}$ and then 40 cycles consisting of $95^{\circ} \mathrm{C}$ for $10 \mathrm{sec}$ and $60^{\circ} \mathrm{C}$ for $35 \mathrm{sec}$. The ABI Prism 7900HT Sequence Detection system (Applied Biosystems, Foster City, CA, USA) was used to analyze the data, and the $\triangle \Delta C T$ method was used to calculate the relative expression of the sample gene (14). The sequences of the primers, which were designed using Primer Premier (v5.0) and were based on the relevant sequences deposited in GenBank, were as follows: rno-miR-29b, 5'-TAGCACCATTTGAAATCAGT GTT-3'; U6, 5'-CAAGGATGACACGCAAATTCG-3'. The relative amount of miR-29b was normalized to that of the U6 RNA.

Transfection of miR-29 mimic or miR-29 inhibitors. miR-29b was either upregulated or downregulated in the NRK-52E cells by transfection with miR-29b mimics or miR-29b inhibitor, respectively. A single-cell suspension was prepared and the cells were cultured in 6-well plates at the density of $1 \times 10^{5}$ cells/well $24 \mathrm{~h}$ prior to transfection. The NRK-52E cells were transfected using Lipofectamine 2000 (Invitrogen) according to the manufacturer's instructions. The cells were 
divided into 5 groups: miR-29b inhibitor group (transfection with miR-29b inhibitor), inhibitor control group (transfection with miRNA synthesized randomly), the blank control group (no transfection), miR-29b mimic group (transfection with miR-29 mimics), mimic control group (transfection with miRNA synthesized randomly). All groups were treated with $100 \mathrm{nM}$ Ang II $24 \mathrm{~h}$ following transfection. The following experiments were carried out after the cells were cultured at $37^{\circ} \mathrm{C}$ in an incubator containing $5 \% \mathrm{CO}_{2}$ for 24 or $48 \mathrm{~h}$.

Dual-luciferase reporter activity assay. miRNA targets were predicted by miRanda (www.microRNA.org) and TargetScan system (https://www.targetscan.org). The wild-type and mutant-type seed region of PIK3R2 (PI3KR2, PI3K regulatory subunit 2 , also known as p85- $\beta$ ) was synthesized and cloned into pMIR-REPORT luciferase vector (Ambion, Inc., Grand Island, NY, USA) Either miR-29b mimic or control were cotransfected with the constructed wild-type or mutanttype luciferase reporter vector into NRK-52E cells using Lipofectamine 2000 (Invitrogen). The cells were harvested $48 \mathrm{~h}$ after transfection and luciferase activity was assessed using the Dual-Luciferase Reporter assay system (Promega, Madison, WI, USA).

Statistical analysis. Statistical analysis was performed by SPSS 20.0 software (IBM Corp., Armonk, NY, USA). The mean values were analyzed for their normality by using the Shapiro-Wilk normality test. All data passed the normality test and were tested for significant difference by one-way analysis of variance, followed by Student's t-test. The data are presented as the mean of SMD. All experiments were performed in triplicate. $\mathrm{P}<0.05$ was considered statistically significant.

\section{Results}

Effects of Ang II on cell morphology. We first assessed the effect of Ang II on cell morphology. Cell morphology was observed by light microscopy after being incubated with $100 \mathrm{nmol} / 1 \mathrm{Ang}$ II for $24 \mathrm{~h}$ (Fig. 1A). The cells in Ang II group showed obvious trans-differentiation with cell polarity disappearing and shape changing from circular to elliptic, suggesting that treatment with the Ang II can induce EMT from the perspective of cell morphology.

Effects of Ang II on EMT molecular marker. Ang II treatment resulted in cell morphology change, so we next examined the change of cell phenotype molecules expressed by NRK-52E after Ang II treatment. By real-time PCR, the mRNA expression level of cytokeratin 18, and vimentin present significant discrepancy between experimental group (Ang II treatment at concentration of $100 \mathrm{nmol} / \mathrm{l}$ for $24 \mathrm{~h}$ ) and control group (Fig. 1B). To detect whether this alteration is time- or dosedependent, the NRK-52E was treated with Ang II (100 nmol/l) for $0,12,24$ and $48 \mathrm{~h}$, respectively. Whilst, another batch of cells were incubated in Ang II with different concentration $(0,10,100$ and $1,000 \mathrm{nmol} / \mathrm{l})$ for $24 \mathrm{~h}$. Cytokeratin 18 showed a time/dose-dependent decrease induced by Ang II in NRK-52E (Fig. 1C and D). In contrast, vimentin showed a time/concentration-dependent increase (Fig. 1C and D).The changes were confirmed by western blotting (Fig. 1E-G).
Effects of Ang II on PI3K/AKT signal pathway and miR-29b. To explore the mechanism underlying the effect of Ang II on PI3K/AKT signal pathway molecules, the PI3K, p-AKT and total AKT were determined in the Ang II group and control group by western blotting. Compared to control group, the expression level of PI3K protein was increased in the Ang II group (Fig. 2A) and the phosphorylation rate of AKT was remarkably elevated (Fig. 2B). Experiments were also conducted to determine the effect of Ang II on miR-29b. After treatment NRK-52E with Ang II (0, 10, 100 and 1,000 nmol/l) for $24 \mathrm{~h}, \mathrm{miR}-29 \mathrm{~b}$ were significantly downregulated by realtime quantitative PCR compared to control (Fig. 2C).

Treatment with PTEN-specific inhibitor SF1670 induced the activation of AKT pathway and occurrence of EMT. To investigate whether the alteration of molecular marker is regulated by PI3K/AKT pathway, the NRK-52E cells were treated with SF1670, a specific inhibitor of PTEN. By inhibiting the activity of endogenous PTEN with SF1670 (Fig. 2D), the AKT pathway was upregulated via increasing the level of cellar PtdIns(3,4,5)P3 (Fig. 2E). Besides, cell phenotype molecules were detected by WB and EMT occurred with the upregulation of AKT pathway by treatment with PTEN-specific inhibitor SF1670 (Fig. 2F). This suggested that EMT is at least partly regulated by $\mathrm{PI} 3 \mathrm{~K} / \mathrm{AKT}$ signal pathway.

Overexpression of miR-29b suppressed phenotype genes involved in EMT and inhibited PI3K and $p$-AKT expression. Treatment with Ang II resulted in EMT-like morphologic changes and the decrease of miR-29b. Then, in order to determined whether overexpression of miR-29b could attune Ang II-induced EMT, NRK-52E were transfected with double-stranded RNA oligonucleotides containing the mature miR-29b sequence (miR-29b mimic), control miRNAs (cont-miR-mimic), miR-29b inhibitor and contmiR-inhibitor. The efficacy of miR-29b transfection was confirmed by real-time RT-PCR (Fig. 3A and B). Introduction of miR-29b significantly reduced protein expression of vimentin (Fig. 3C and D), and increased protein expression of cytokeratin 18 (Fig. 3C and E). In contrast, low expression of miR-29b by transfecting miR-29b inhibitor resulted in the increased expression of vimentin (Fig. 3C and D) and decreased expression of cytokeratin 18 (Fig. 3C and E). Lastly, to further assess the effect of miR-29b on PI3K/AKT, the protein expression of PI3K, and p-AKT were examined by western blot analysis after transfection of miR-29b-3p. As shown in Fig. 3F and G, Ang II-treated cells transfected with miR-29b mimic showed significant reduced expression of PI3K and p-AKT compared to cont-miR-mimic or untreated cells. In contrast, downregulation of miR-29b by transfected miR-29b inhibitor resulted in the upregulation of PI3K/AKT pathway.

miR-29b inhibits PI3K and AKT expression by directly binding to their 3'-UTR regions. The above data suggested PI3K, AKT,vimentin,cytokeratin 18 were regulated by miR29 in NRK-52E. However, the regulation could be indirect. To investigate whether miR-29b directly targets PI3K, AKT, vimentin, cytokeratin 18, we searched for the targets of miR-29b using computational prediction analysis in several miRNA target 


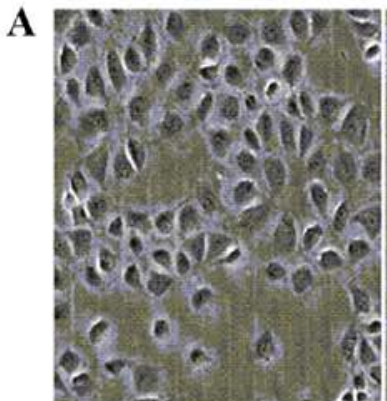

Control group

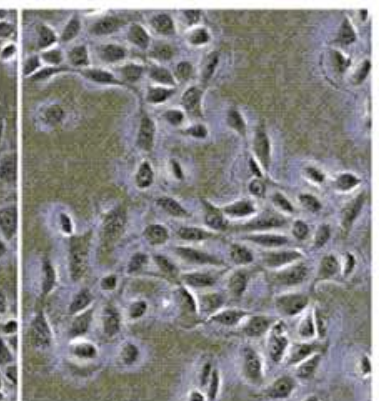

Treatment group
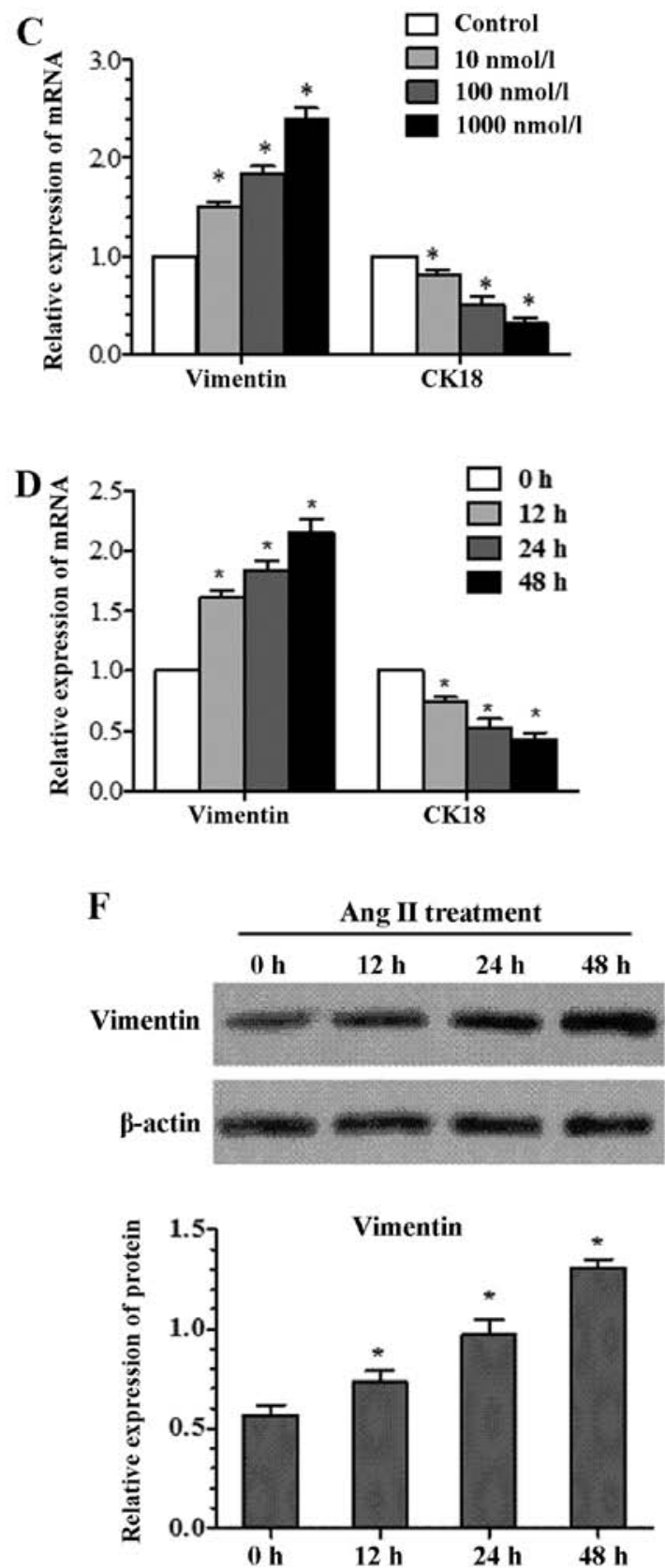

B

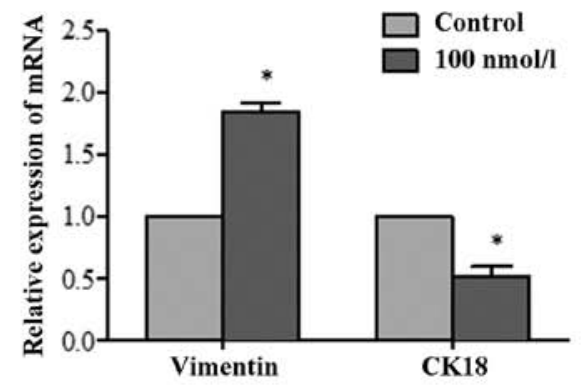

$\mathbf{E}$ Ang II treatment
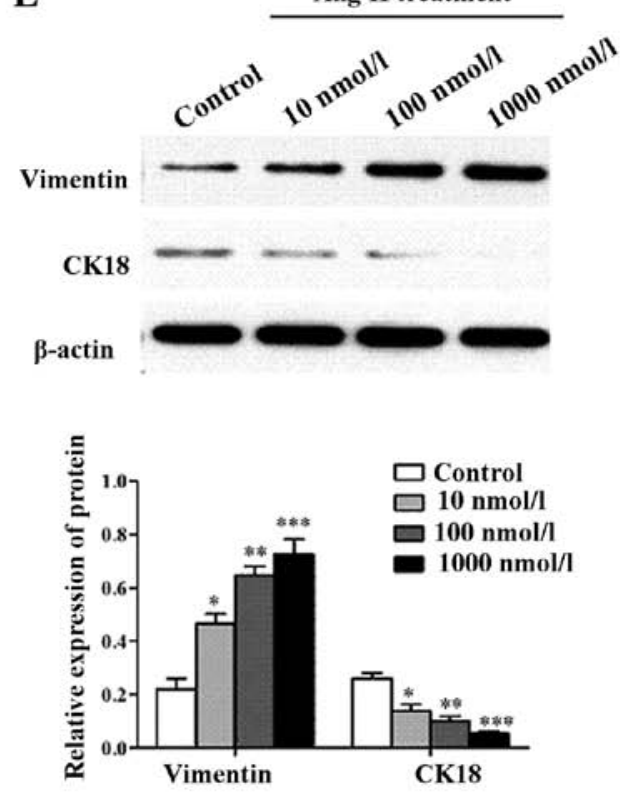

G
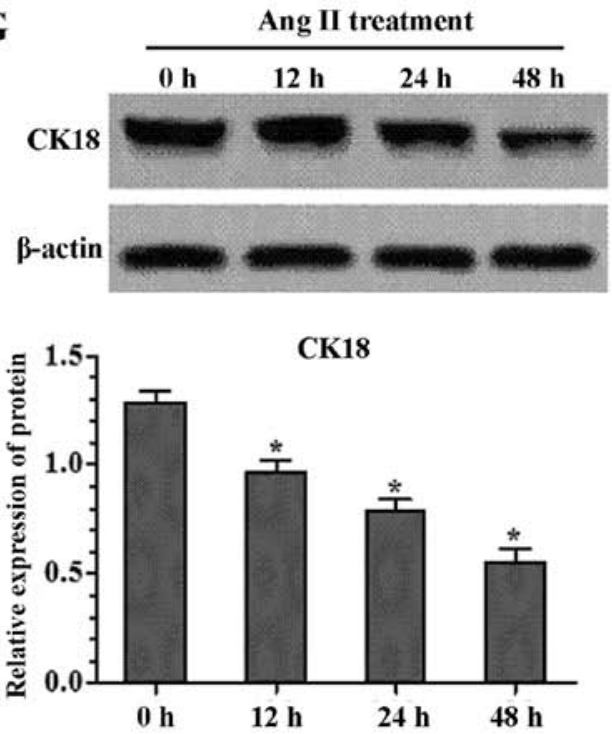

Figure 1. Effects of angiotensin II (Ang II) on cell morphology and epithelial-mesenchymal transition (EMT) molecular marker. (A) The cells in Ang II group showed obvious trans-differentiation with cell polarity disappearing and shape changing from circular to elliptic (magnification, $\mathrm{x} 400$ ). (B) NRK-52E cells were cultured in the presence of Ang II (100 nmol/1 for $24 \mathrm{~h})$. After treatment with Ang II, vimentin gene was significanttly increased by real-time quantitative PCR, whereas cytokeratin 18 expression was significant decreased. (C) The expression of cytokeratin 18 and vimentin as assessed by real-time quantitative PCR, showed significant alteration after treatment NRK-52E with Ang II (0, 10, 100 and 1,000 nmol/l) for 24 h. (D) Treatment NRK-52E with Ang II $(100 \mathrm{nmol} / \mathrm{l})$ for $0,12,24$ and $48 \mathrm{~h}$, resulted in a reduction in cytokeratin 18 expression and increase in vimentin expression as assessed by real-time quantitative PCR ("P<0.05 compared with control). (E) Western blotting revealed a similar change in cytokeratin 18 and vimentin protein expression after NRK-52E treatment with Ang II in doses 0, 10, 100 and 1,000 nmol/l for $24 \mathrm{~h}$. After treatment with Ang II (100 nmol/l) for 0, 12, 24 and $48 \mathrm{~h},(\mathrm{~F})$ vimentin protein was significantly increased by western blotting, $(\mathrm{G})$ whereas cytokeratin 18 protein expression was significantly decreased (" $\mathrm{P}<0.05$ compared with control). 

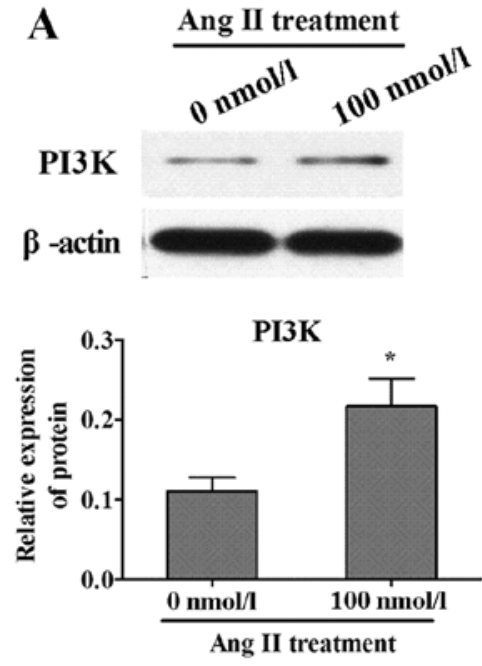

D

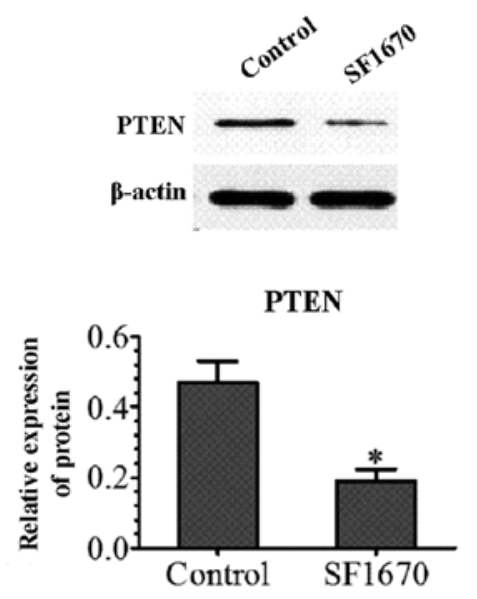

B
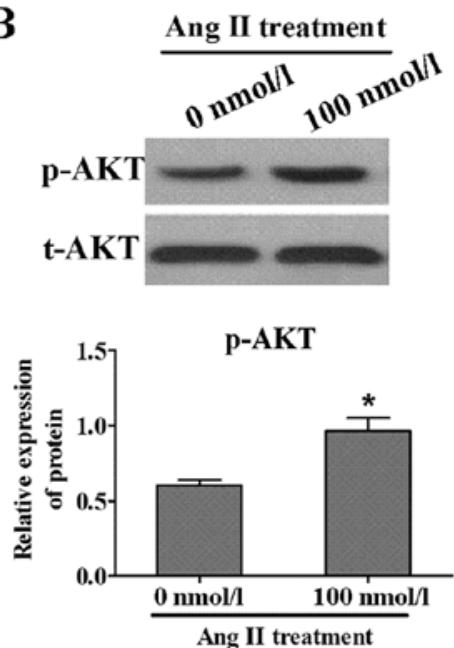

$\mathbf{E}$
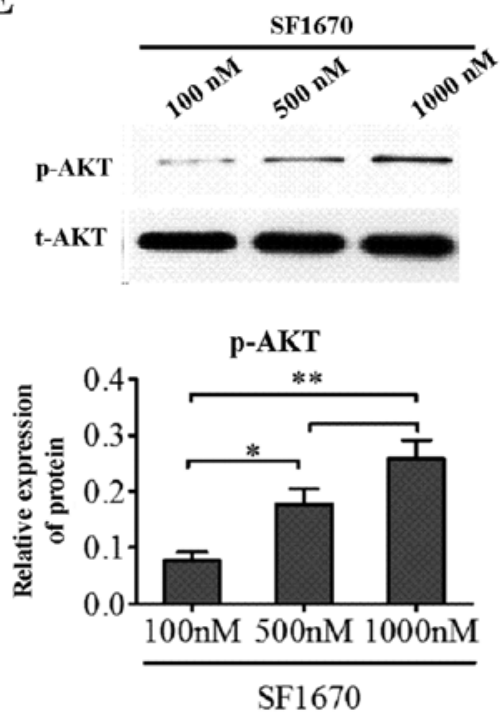

$\mathrm{C}$

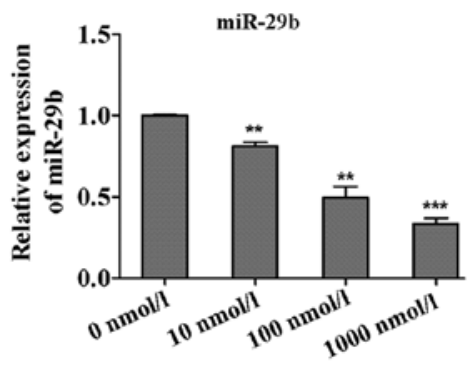

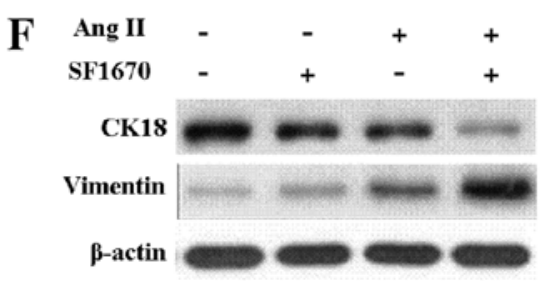

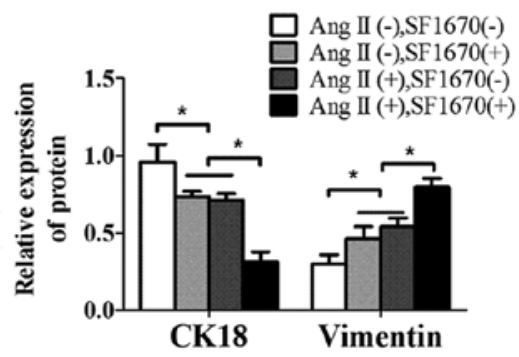

Figure 2. Effects of angiotensin II (Ang II) on phosphatidylinositol 3-kinase/protein kinase B (PI3K/AKT) signal pathway and miR-29b. NRK-52E cells were cultured in the presence of Ang II (100 nmol/1 for $24 \mathrm{~h})$. After treatment with Ang II, (A) PI3K protein level was significantly increased by western blotting, (B) as well as the phosphorylation rate of AKT. (C) Real-time quantitative PCR were conducted to examine the expression of miR-29b. Treatment with 0 nmol/1 Ang II were used as a control ( ${ }^{*} \mathrm{P}<0.05$ compared with control). Activation of AKT pathway can induce the occurrence of epithelial-mesenchymal transition (EMT) in NRK-52E. NRK-52E cells were incubated with SF1670 for $1 \mathrm{~h}$. Western blotting were conducted to examine the expression of (D) phosphatase and tensin homolog on chromosome 10 (PTEN) and (E) p-AKT. (F) Besides, the vimentin and CK18 protein were also detected by western blotting in the absence/ presence of Ang II or/and SF1670, ${ }^{*} \mathrm{P}<0.05$ compared with control.

databases including TargetScan, Scan6.2, miRANDA and miRDB. We found that miR-29b has one putative target site in the 3'-UTR of PIK3R2 (PI3KR2, PI3K regulatory subunit 2, also known as p85- $\beta$ ) (Fig. $3 \mathrm{H}$ ), but not AKT1, vimentin and cytokeratin 18 . Moreover, these target site sequences are highly conserved among human, mouse and rat, demonstrating that miR-29b may act as a direct regulator of PIK3R2. To validate whether the putative miR-29b target sequence in 3'-UTR of PIK3R2 directly regulate gene expression, we constructed pMIR report plasmids encoding a firefly luciferase transcript with either wild-type or mutant 3'-UTR of PIK3R2 (wt-PIK3R2 and mut-PIK3R2). We evaluated their respective luciferase reporter activity after co-transfection with miR-29b mimic or cont-miR-mimic in NRK-52E cells. The results showed that miR-29b mimic repressed the reporter activity of the transcript containing wild-type 3'-UTR of PIK3R2 (Fig. 3I), suggesting that PIK3R2 is definite target of miR-29b.

\section{Discussion}

The important role of miRNAs as regulators of physiologic and pathologic processes in human health and disease has recently been recognized. The signal transduction pathway within cells is a highly complex process mediating cell survival, differentiation and metabolism. In this study, we demonstrated the role of PI3K/AKT pathway that miR-29b plays in the regulation of EMT induced by treatment of Ang II in NRK-52E.

Angiotensin II (Ang II) has been proven to plays an important role in the occurrence and development of hypertension, as well as in EMT $(9,15)$, which is a crucial step in the development of renal fibrosis. EMT is characterized by a phenotypic transformation from epithelial cells to a fibroblast-like morphology. During this process, there is acquisition of mesenchymal markers, such as $\alpha$-SMA and vimentin (16), and a loss of epithelium markers, including 
A
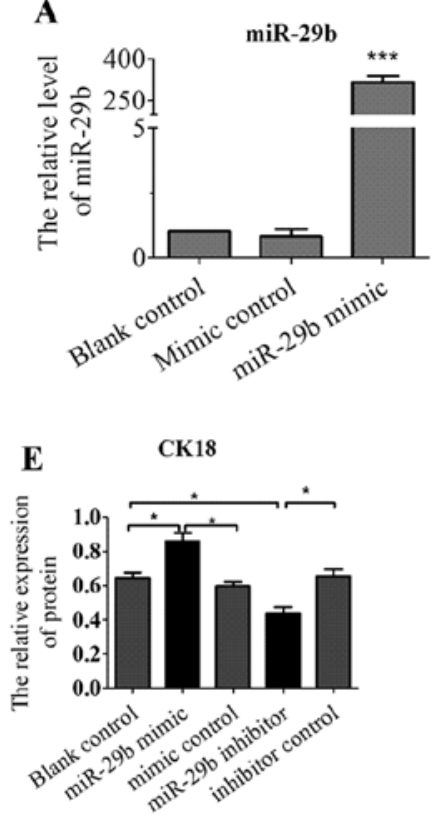

F

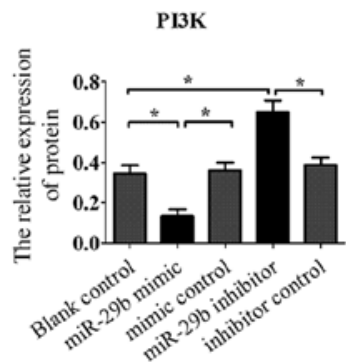

B

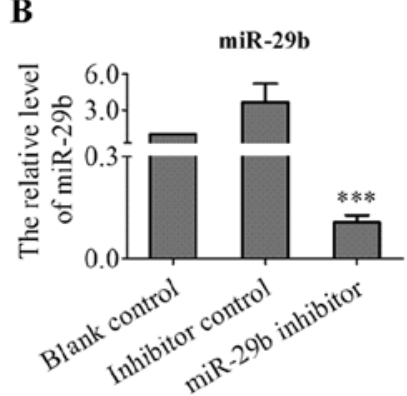

G

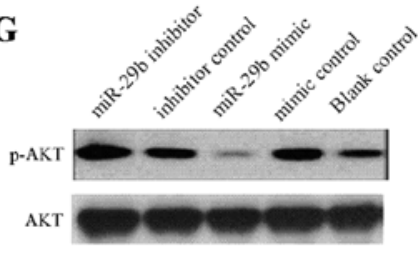

p-AKT

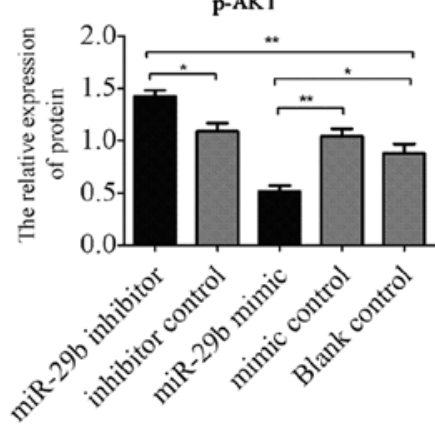

C
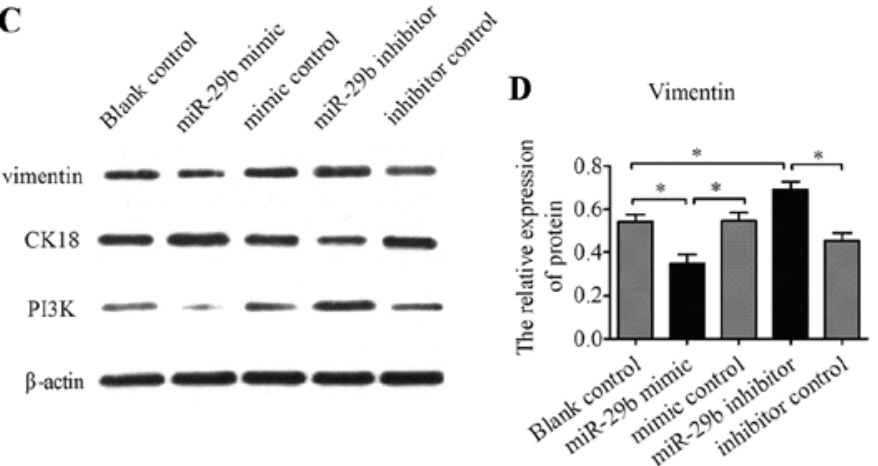

$\mathbf{H}$

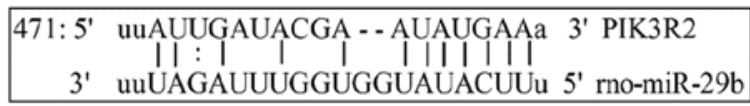

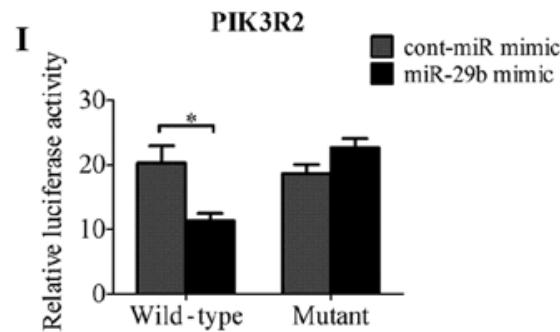

Figure 3. Overexpression of miR-29b alleviates the epithelial-mesenchymal transition (EMT) and inhibits PI3K and p-AKT expression. NRK-52E cells were incubated with angiotensin II (Ang II) $(100 \mathrm{nmol} / \mathrm{l})$ for $24 \mathrm{~h}$ after transfection. (A and B) Real-time quantitative PCR were conducted to examine the level of miR-29b after trancfection. (C and D) Vimentin and (C and E) cytokeratin 18 expression was determined by western blotting. (C and F) PI3K and (G) p-AKT expression were also detected by western blotting. ${ }^{\mathrm{P}}<0.05$ compared with control. miR-29b inhibits PI3K and AKT expression by directly binding to their 3'-untranslated region (3'-UTR). (H) Binding of miR-29b with PIK3R2 3'-UTR was predicted by TargetScan. (I) Dual luciferase reporter gene assay revealed that miR-29b significantly decreased the luciferase activity of PIK3R2 wild-type (wt) 3'-UTR.

E-cadherin and cytokeratin 18 (17), which is essential for the structural integrity of the renal epithelium. In this study, we found a significant decrease of cytokeratin 18 and increase of vimentin in a concentration-dependent manner after treatment of NRK-52E with Ang II. The change of cell morphology and phenotype molecules observed in the study indicated that the cell underwent a epithelial-to-mesenchymal transformation of tubular cells into a myofibroblastic phenotype. These findings are consistent with the previous study $(9,14,18,19)$. In addition, treatment of NRK-52E with Ang II resulted in the significant upregulation of PI3K/AKT signal pathway just as reported by Yano et al (20) that Ang II can activate the PI3K/AKT signal pathway through binding to AT1R. Besides, inhibiting PTEN with SF1670 upregulated the level of p-AKT and induced the occurrence of EMT which was also found following treatment of Ang II.

Together, these findings indicated that Ang II can induce EMT through the signal pathway of PI3K/AKT in NRK-52E (Fig. 4A). As a main regulator of renal profibrotic factors (21), the traditional roles of Ang II in the induction of EMT are mainly mediated by directly activating TGF- $\beta /$ Smad signalling pathway (22-24), or by indirectly activating TGF- $\beta /$ Smad signaling via an ERK/p38 MAP
kinase-Smad crosstalk mechanism (25). This study provided new insight into the intracellular signal transduction involved in the EMT induced by Ang II treatment.

Although the evidence suggesting a role for miRNAs as biomarkers and novel targets for treatment in kidney disease has been reported (26-28), the effect of known nephroprotective treatments on the expression of miRNAs, or their possible contribution as protective agents against renal fibrosis, has yet to be fully established. In this context, we also evaluated miR-29b expression in the Ang II-treated cells and we found that a significant difference was displayed in the level of miR-29b compared to the control group. Treatment with Ang II resulting in a marked decrease in miR-29b expression apart from upregulation of p-AKT and occurrence of EMT demonstrated that miR-29b may be involved in PI3K/AKT signal pathway and regulate the EMT of NRK-52E (Fig. 4B). This finding may perfect the hypothesis of Pan et al (15) that at least two distinct intracellular signaling pathways are involved in the low levels of miR-29b expression after treatment of Ang II in NRK-52E.

If so, it would be important to establish that its overexpression attenuated the severity of Ang II-induced EMT via PI3K/AKT signal pathway. To confirm this, NRK-52E cells 


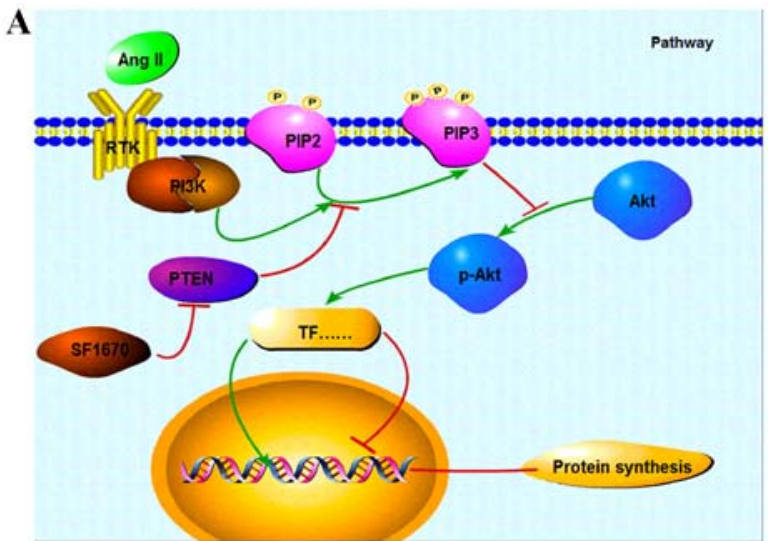

B

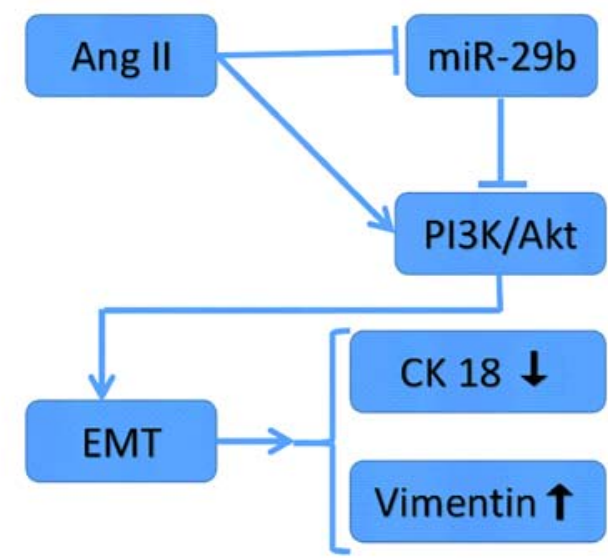

Figure 4. Phosphatidylinositol 3-kinase/protein kinase B (PI3K/AKT) pathway. (A) Angiotensin II (Ang II) can induce epithelial-mesenchymal transition (EMT) in NRK-52E via the activation of PI3K/AKT pathway, which can be inhibited by phosphatase and tensin homolog on chromosome 10 (PTEN). SF1670 can also induce similar change to Ang II. (B) miR-29b may ameliorate Ang II-induced EMT by the suppression of PI3K/AKT signaling. RTK, receptor tyrosine kinase; $\mathrm{TF}$, transcription factors.

were transfected with miR-29b mimics and then treated with Ang II for $24 \mathrm{~h}$. The result of western blot analysis and immunoflorescence staining demonstrated that overexpression of miR-29b displayed downregulation of PI3K/AKT signal pathway and suppressed the Ang II-induced upregulation of vimentin and downregulation of cytokeratin 18 . These results manifest that the overexpression of miR-29b negatively modulates EMT by downregulation of PI3K/AKT signal pathway. Moreover, transfection of NRK-52E with miR-29b inhibitor was also conducted. Western blot analysis and immunoflorescence staining revealed that miR-29b inhibitor induced a marked increase of vimentin and decrease of cytokeratin 18 as well as downregulation of PI3K/AKT signal pathway. The data indicated that the treatment with Ang II can lower the level of miR-29b, activate the PI3K/AKT signal pathway and eventually trigger the occurrence of EMT,which can be negatively modulated by overexpresson of miR-29b.

In conclusion, Ang II can induce EMT in NRK-52E via the activation of PI3K/AKT signaling and elevated expression of miR-29b may ameliorate Ang II-induced EMT by the suppression of PI3K/AKT signaling. These results offer the possibility that specific inhibitor of PI3K/AKT cascade or enhancing miR-29b level by gene therapy may have beneficial effects in attenuating renal interstitial fibrosis.

\section{Acknowledgements}

The research was supported by the Research Center of Zhongnan Hospital of Wuhan University.

\section{Funding}

This study was supported by the Key Foundation of Hubei Nature Scientific Funds (grant no. 2013CFB227).

\section{Availability of data and material}

The datasets used and/or analyzed during the current study are available from the corresponding author on reasonable request.

\section{Authors' contributions}

HH and HS conceived and designed the study. HH, SH, SX, YG and FZ performed the experiments. HH wrote the paper. $\mathrm{SH}, \mathrm{SX}, \mathrm{YG}$ and FZ reviewed and edited the manuscript. All authors read and approved the final manuscript.

\section{Ethics approval and consent to participate}

Not applicable.

\section{Consent for publication}

Not applicable.

\section{Competing interests}

The authors declare that they have no competing interests.

\section{References}

1. Ballhause TM, Soldati R and Mertens PR: Sources of myofibroblasts in kidney fibrosis: All answers are correct, however to different extent! Int Urol Nephrol 46: 659-664, 2014.

2. Liu Y: New insights into epithelial-mesenchymal transition in kidney fibrosis. J Am Soc Nephrol 21: 212-222, 2010.

3. Cantrell DA: Phosphoinositide 3-kinase signalling pathways. J Cell Sci 114: 1439-1445, 2001.

4. Ye B, Jiang LL, Xu HT, Zhou DW and Li ZS: Expression of PI3K/AKT pathway in gastric cancer and its blockade suppresses tumor growth and metastasis. Int J Immunopathol Pharmacol 25: 627-636, 2012.

5. Hassan B, Akcakanat A, Holder AM and Meric-Bernstam F: Targeting the PI3-kinase/Akt/mTOR signaling pathway. Surg Oncol Clin N Am 22: 641-664, 2013.

6. Yang T, Liang Y, Lin Q, Liu J, Luo F, Li X, Zhou H, Zhuang S and Zhang H: miR-29 mediates TGF $\beta 1$-induced extracellular matrix synthesis through activation of PI3K-AKT pathway in human lung fibroblasts. J Cell Biochem 114: 1336-1342, 2013.

7. Wang J, Chu ES, Chen HY, Man K, Go MY, Huang XR, Lan HY, Sung JJ and Yu J: microRNA-29b prevents liver fibrosis by attenuating hepatic stellate cell activation and inducing apoptosis through targeting PI3K/AKT pathway. Oncotarget 6: 7325-7338, 2015.

8. Qin J, Xie YY, Huang L, Yuan QJ, Mei WJ, Yuan XN, Hu GY, Cheng GJ, Tao LJ and Peng ZZ: Fluorofenidone inhibits nicotinamide adeninedinucleotide phosphate oxidase via PI3K/Akt pathway in the pathogenesis of renal interstitial fibrosis. Nephrology (Carlton) 18: 690-699, 2013.

9. Burns WC, Velkoska E, Dean R, Burrell LM and Thomas MC: Angiotensin II mediates epithelial-to-mesenchymal transformation in tubular cells by ANG 1-7/MAS-1-dependent pathways. Am J Physiol Renal Physiol 299: F585-F593, 2010. 
10. Yan B, Guo Q, Fu FJ, Wang Z, Yin Z, Wei YB and Yang JR: The role of miR-29b in cancer: Regulation, function, and signaling. Onco Targets Ther 8: 539-548, 2015.

11. Bayoumi AS, Sayed A, Broskova Z, Teoh JP, Wilson J, Su H Tang YL and Kim IM:Crosstalk between long noncoding RNAs and microRNAs in health and disease. Int J Mol Sci 17: E356, 2016.

12. van Rooij E, Sutherland LB, Thatcher JE, DiMaio JM, Naseem RH, Marshall WS, Hill JA and Olson EN: Dysregulation of microRNAs after myocardial infarction reveals a role of miR-29 in cardiac fibrosis. Proc Natl Acad Sci USA 105: 13027-13032, 2008

13. Cushing L, Kuang PP, Qian J, Shao F, Wu J,Little F, Thannickal VJ, Cardoso WV and Lü J: miR-29 is a major regulator of genes associated with pulmonary fibrosis. Am J Respir Cell Mol Biol 45: 287-294, 2011.

14. Livak KJ and Schmittgen TD: Analysis of relative gene expression data using real-time quantitative PCR and the 2(-Delta Delta C(T)) method. Methods 25: 402-408, 2001.

15. Pan J, Zhang J, Zhang X, Zhou X, Lu S, Huang X, Shao J, Lou G, Yang D and Geng YJ: Role of microRNA-29b in angiotensin II-induced epithelial-mesenchymal transition in renal tubular epithelial cells. Int J Mol Med 34: 1381-1387, 2014.

16. Okada H, Ban S, Nagao S, Takahashi H, Suzuki H and Neilson EG: Progressive renal fibrosis in murine polycystic kidney disease: An immunohistochemical observation. Kidney Int 58: 587-597, 2000

17. Djudjaj S, Papasotiriou M, Bülow RD, Wagnerova A Lindenmeyer MT, Cohen CD, Strnad P, Goumenos DS, Floege J and Boor P: Keratins are novel markers of renal epithelial cell injury. Kidney Int 89: 792-808, 2016.

18. Chen J, Chen JK and Harris RC: Angiotensin II induces epithelialto-mesenchymal transition in renal epithelial cells through reactive oxygen species/Src/caveolin-mediated activation of an epidermal growth factor receptor-extracellular signal-regulated kinase signaling pathway. Mol Cell Biol 32: 981-991, 2012.
19. Burns WC and Thomas MC: Angiotensin II and its role in tubular epithelial to mesenchymal transition associated with chronic kidney disease. Cells Tissues Organs 193: 74-84, 2011.

20. Yano N, Suzuki D, Endoh M, Zhao TC, Padbury JF and Tseng YT: A novel phosphoinositide 3-kinase-dependent pathway for angiotensin II/AT-1 receptor-mediated induction of collagen synthesis in MES-13 mesangial cells. J Biol Chem 282: 18819-18830, 2007.

21. Macconi D, Remuzzi G and Benigni A: Key fibrogenic mediators: Old players. Renin-angiotensin system. Kidney Int Suppl (2011) 4: 58-64, 2014.

22. Zavadil J and Böttinger EP: TGF-beta and epithelial-to-mesenchymal transitions. Oncogene 24: 5764-5774, 2005.

23. Yang F, Huang XR, Chung AC, Hou CC, Lai KN and Lan HY: Essential role for Smad3 in angiotensin II-induced tubular epithelial-mesenchymal transition. J Pathol 221: 390-401, 2010.

24. Meng XM, Tang PM, Li J and Lan HY: TGF- $\beta /$ Smad signaling in renal fibrosis. Front Physiol 6: 82, 2015.

25. Rodríguez-Vita J, Sánchez-López E, Esteban V, Rupérez M, Egido J and Ruiz-Ortega M: Angiotensin II activates the Smad pathway in vascular smooth muscle cells by a transforming growth factor-beta-independent mechanism. Circulation 111: 2509-2517, 2005.

26. Denby L and Baker AH: Targeting non-coding RNA for the therapy of renal disease. Curr Opin Pharmacol 27: 70-77, 2016.

27. Simpson K, Wonnacott A, Fraser DJ and Bowen T: MicroRNAs in diabetic nephropathy: From biomarkers to therapy. Curr Diab Rep 16: 35, 2016

28. Gomez IG, Nakagawa N and Duffield JS: MicroRNAs as novel therapeutic targets to treat kidney injury and fibrosis. Am J Physiol Renal Physiol 310: F931-F944. 